\title{
Poncelet-type Problems, an Elementary Approach
}

András Hraskó

András Hraskó studied maths in Budapest at Eötvös Loránd University. He obtained his Msc in Warwick. Since 1991 he has been teaching mathematics in grammar school. He is now preparing his PhD. In his free time he hikes in mountains.

\section{Introduction}

Certain recursive geometric processes sometimes become periodic. As a rule, periodicities of this type reveal beautiful geometric configurations and often lead to remarkable theorems.

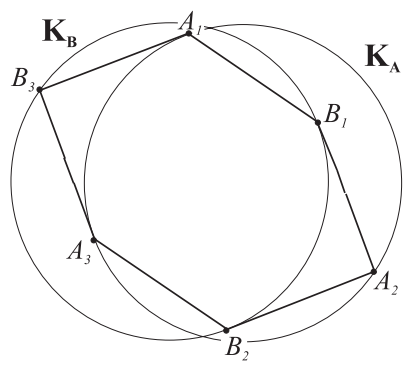

Fig. 1

The aim of the present work is to put forward pictures and statements of this kind through specific problems and their solutions and in this rather elementary way to call the reader's

Der Inhalt von Schliessungssätzen der Geometrie, des Satzes von Desargues, des Satzes von Pappus, des Satzes von Poncelet und von anderen, hat jeweils etwas Geheimnisvolles an sich: Welche Magie erzwingt, dass sich eine nach komplizierten Vorschriften gebildete Figur am Ende schliesst? Man staunt, man wundert sich. Die Mathematik ist aufgerufen, die Ursachen zu ergründen und zu erklären, den Satz zu beweisen. - András Hraskó bespricht in seinem Beitrag in elementarer Weise eine Reihe derartiger Sätze - man könnte sie alle Zig-Zag-Sätze nennen -, die auf zunächst verborgene Weise mit dem Poncelet-Theorem zusammenhängen. ust 
attention to the theorem of Poncelet, lying behind all of these periodic structures. In our day this fascinating theorem has become fashionable again as is clearly demonstrated by the number of excellent recent publications on the subject. The present work may serve as an introduction to the study of these works.

In the first three sections three different types of problems are considered while Section 4 is devoted to the interrelation between them.

\section{Zig-zags}

Problem 1. (Generalization of the construction of the regular hexagon) (Fig. 1)

Given two circles $\mathbf{K}_{\mathbf{A}}$ and $\mathbf{K}_{\mathbf{B}}$ of radius $\rho$ neither of which contains the centre of the other, and the points $A_{1}$ and $B_{1}$ at a distance $\rho$ from each other on the circles $\mathbf{K}_{\mathbf{A}}, \mathbf{K}_{\mathbf{B}}$ respectively. Construct the equilateral polygon $A_{1} B_{1} A_{2} B_{2} \cdots$ according to the following recursion which will be called the Zig-zag process:

1) Let $A_{n+1}$ be that point on $\mathbf{K}_{\mathbf{A}}$ which is at a distance $\rho$ from $B_{n}$ and which is different from $A_{n}$. If no such point exists then put $A_{n+1}=A_{n}$.

2) Let $B_{n+1}$ be that point on $\mathbf{K}_{\mathbf{B}}$ which is at a distance $\rho$ from $A_{n}$ and which is different from $B_{n}$. If no such point exists then put $B_{n+1}=B_{n}$.

Prove that $A_{4}=A_{1}$ !

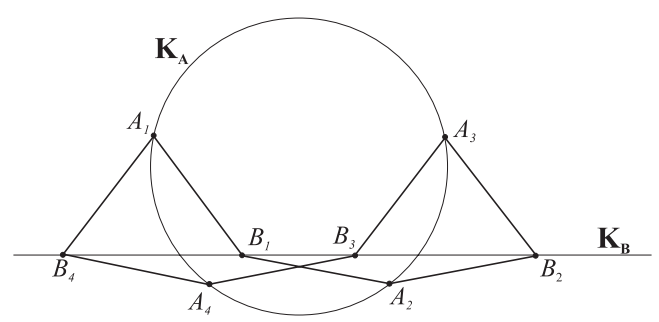

Fig. 2

Problem 2. (Fig. 2)

Let $\mathbf{K}_{\mathbf{A}}$ be a given circle of radius $\rho$, the straight line $\mathbf{K}_{\mathbf{B}}$, not going through the centre of $\mathbf{K}_{\mathbf{A}}$, and the points $A_{1}$ and $B_{1}$ on $\mathbf{K}_{\mathbf{A}}$ and $\mathbf{K}_{\mathbf{B}}$ respectively which are at a distance $\rho$ from each other.

Construct the equilateral polygon $A_{1} B_{1} A_{2} B_{2} \cdots$ according to the Zig-zag process of the previous problem.

Prove that $A_{5}=A_{1}$ !

These two problems are deduced from the general formulae to be discussed in Section 4. Problem 3 - or rather its generalization - is not a new one and has already been thoroughly discussed in [17] (see also [18] and [19]). 


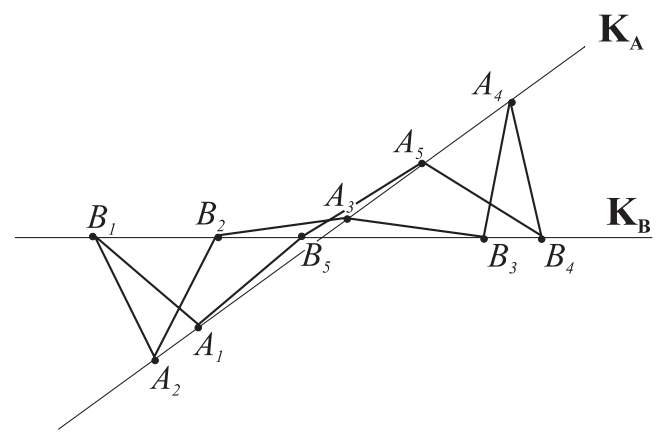

Fig. 3

Problem 3. (Fig. 3)

Given the straight lines $\mathbf{K}_{\mathbf{A}}$ and $\mathbf{K}_{\mathbf{B}}$ which intersect at an angle $36^{\circ}$, and points $A_{1}$ and $B_{1}$ on $\mathbf{K}_{\mathbf{A}}$ and $\mathbf{K}_{\mathbf{B}}$ respectively. Construct the equilateral polygon $A_{1} B_{1} A_{2} B_{2} \cdots$ of arbitrary side length by means of the Zig-zag process.

Prove that $A_{6}=A_{1}$ !

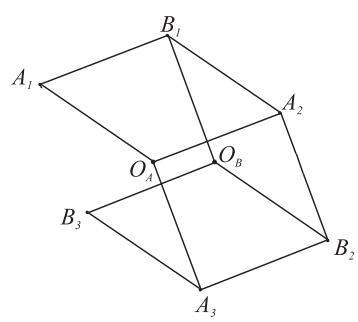

Fig. 4

Solution to Problem 1.

In Fig. 4 instead of $\mathbf{K}_{\mathbf{A}}$ and $\mathbf{K}_{\mathbf{B}}$ only their centres $O_{A}, O_{B}$ and the radii connecting them with the polygon's vertices are shown. All the segments, appearing in the figure are of length $\rho$. One has to prove that the segment $B_{3} A_{1}$ is also of length $\rho$.

All the segments $A_{1} B_{1}, O_{A} A_{2}, A_{3} B_{2}, B_{3} O_{B}$ are parallel to each other since the quadrilaterals are all rhombi. Therefore, $A_{1} B_{1} O_{B} B_{3}$ is also a parallelogram and the side $A_{1} B_{3}$ together with the opposite side $B_{1} O_{B}$ are of length $\rho$.

In Fig. 4 a cube is clearly recognizable. A great many of elementary problems lead to the same configuration.

\section{Solution to Problem 2.}

In Fig. $5 O_{A}$ denotes the centre of the circle $\mathbf{K}_{\mathbf{A}}$. All the segments shown on the figure are of length $\rho$. The segments $A_{1} B_{1}, O_{A} A_{2}, A_{3} B_{2}$ are equal and parallel since quadrilaterals $A_{1} B_{1} A_{2} O_{A}, A_{2} O_{A} A_{3} B_{2}$ are rhombi. Hence, the quadrilateral $A_{1} B_{1} B_{2} A_{3}$ is a parallelogram too and, therefore, $A_{1} A_{3}$ is parallel to the straight line $\mathbf{K}_{\mathbf{B}}$. If one 
increases the indices in the proof of Problem 1 by 2, one arrives at the conclusion that $A_{3} A_{5}$ is parallel to $\mathbf{K}_{\mathbf{B}}$ and, as a consequence, $A_{5}$ does indeed coincide with $A_{1}$.

In Fig. 5 two half-cubes can now be recognized.

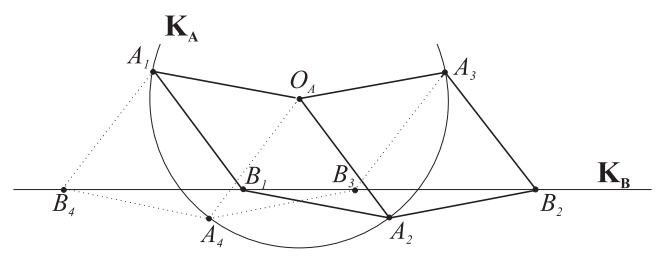

Fig. 5

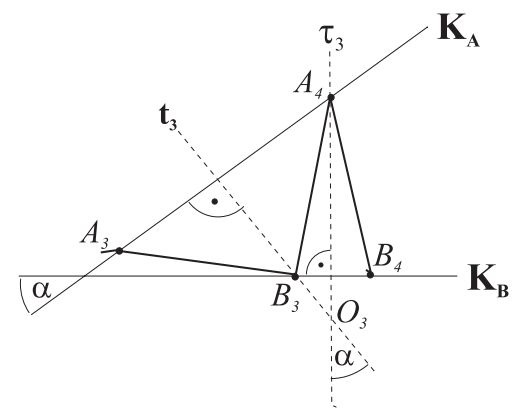

Fig. 6

Solution to Problem 3.

This problem is a bit more difficult than the previous ones but its generalization is more straightforward than that of those. Below, in discussing the "recurrence problem", we allow the straight lines $\mathbf{K}_{\mathbf{A}}$ and $\mathbf{K}_{\mathbf{B}}$ to cross at an arbitrary angle rather than at $36^{\circ}$. We choose here an approach different from that of [17] closely connected to the recent proofs of the rather more difficult theorem of Poncelet ([10], [12], [20], [21]), although the proof in [17] has the merit of revealing the polyhedron hidden behind the configuration.

In Fig. 6 part of the configuration, the portion $A_{3} B_{3} A_{4} B_{4}$ of the polygon, is seen. We are to look for the transformation which maps the segment $A_{3} B_{3}$ onto $A_{4} B_{4}$.

Consider the reflections with respect to the perpendicular bisector $\mathbf{t}_{3}$ of segment $A_{3} A_{4}$ and the perpendicular bisector $\tau_{3}$ of $B_{3} B_{4}$. Under the first of these transformations $A_{3} B_{3}$ is taken to $A_{4} B_{3}$ while the second one accomplishes the transformation of $A_{3} B_{3}$ onto $A_{4} B_{4}$. The desired transformation is, therefore, the composition of two reflections, which is a rotation around the point of intersection of the two axes of reflection. The angle of rotation is twice the angle of the axes $\mathbf{t}_{3}, \tau_{3}$ i.e. $2 \alpha$.

In more general terms we can say that the segment $A_{n+1} B_{n+1}$ can be obtained from $A_{1} B_{1}$ by an $A_{1} B_{1}$ dependent transformation $\varphi_{n}$ which is the composition of rotations around 
a series of points $O_{1}, O_{2}, \cdots O_{n}$, by the same angle $2 \alpha$. The composition of rotations is also a rotation, with a single exception: when the sum of the rotation angles is a multiple of $360^{\circ}$, the composition becomes a translation or the identity transformation.

In our case translation is out of the question. When $A_{1} \in \mathbf{K}_{\mathbf{A}}, B_{1} \in \mathbf{K}_{\mathbf{B}}$ and the straight lines $\mathbf{K}_{\mathbf{A}}, \mathbf{K}_{\mathbf{B}}$ are not parallel, no translation of the segment $A_{1} B_{1}$ exists for which $A_{1}$ and $B_{1}$ remain on $\mathbf{K}_{\mathbf{A}}$ and $\mathbf{K}_{\mathbf{B}}$ respectively.

If, therefore, $2 \alpha \cdot n=k \cdot 360^{\circ}$, then $\varphi_{n}$ is the identity: $A_{n+1}=A_{1}, B_{n+1}=B_{1}$. The case $\alpha=36^{\circ}$ assumed in Problem 3 corresponds to $n=5, k=1$.

If, on the other hand, $2 \alpha \cdot n \neq k \cdot 360^{\circ}$ then $\varphi_{n}$ is a generic rotation. Since it has only a single fixed point, $\varphi_{n}\left(A_{1} B_{1}\right)$ must be different from $A_{n} B_{n}$.

There exists a general theorem hidden in the background of the three problems discussed so far:

The Zig-zag theorem. Let both the objects $\mathbf{K}_{\mathbf{A}}$ and $\mathbf{K}_{\mathbf{B}}$ be either a circle or a straight line in space. A fixed length $\rho$ is also given and it is assumed that neither object has a point from which all the points of the other are at a distance $\rho^{1)}$. If a Zig-zag process, starting from some given segment $A_{1} B_{1}$ of length $\rho\left(A_{1} \in \mathbf{K}_{\mathbf{A}}, B_{1} \in \mathbf{K}_{\mathbf{B}}\right)$ is of periodicity $n$ (i.e. $\left.B_{n+1}=B_{1}\right)$, then any other initial segment of the same length $\rho$ gives rise to a process of the same periodicity $n$.

This theorem was discovered by Black W.L., Howland H.C. and Howland B. for the case when both objects are circles, and in their 1974 paper [15] they gave an elegant analytic proof of it. Since then several different proofs have been published ([20], [23]). However, as stated by Black and the Howlands, this beautiful theorem is of existential character, since no hint is given of the conditions the circles and the length $\rho$ must satisfy in order to give rise to an $n$-step periodic Zig-zag configuration (which from now on we will denote by $Z_{n}$ ). The aim of the present work is to make progress toward the detailed characterization of $Z_{n}$-configurations.

Let us introduce the simpler notation $\mathbf{K}, \mathbf{L}$ for the objects $\mathbf{K}_{\mathbf{A}}, \mathbf{K}_{\mathbf{B}}$. For simplicity's sake we confine ourselves to the case when $\mathbf{K}$ and $\mathbf{L}$ are circles which lie in the same plane. In this case the relevant parameters of the configuration are the radii $r$ and $R$ of $\mathbf{K}$ and $\mathbf{L}$, the distance $d$ between their centres and the length $\rho$ of the "zig" (and "zag" of course). In this particular case the Zig-zag configuration will be denoted by $\mathrm{Z}(r, R, d, \rho)$.

One may suspect that the geometrical condition of $n$-step periodicity must have an analytic counterpart, a function $z_{n}$ of four variables, perhaps a polynomial, which vanishes iff the configuration is $Z_{n}$ :

$$
z_{n}(r, R, d, \rho)=0 \Longleftrightarrow Z(r, R, d, \rho) \text { form a } Z_{n} \text { configuration. }
$$

If one of the objects, say $\mathbf{L}$, is a straight line, then the system is defined by the radius $r$ of $\mathbf{K}$, the distance $\Delta$ of its centre from $\mathbf{L}$ and, of course, $\rho$. This configuration will be

1) This condition serves to ensure the uniqueness of the Zig-zag process. 
denoted by $Z^{\prime}(r, \Delta, \rho)$. In this case the function $z_{n}^{\prime}$ the zeros of which correspond to the $n$-step periodicity of the configuration depends only on three variables.

The rather long route to the functions $z_{n}, z_{n}^{\prime}$ will be described in Section 4 . They turn out to be polynomials indeed. The configurations of Problem 1 and 2 originate in the explicit form of these polynomials.

\section{Polygons inscribed in a circle whose midpoints are also on some circle}

Problem 4. Given the circles $\mathbf{K}$ and $\mathbf{L}$ on the plane, construct

a) the triangle (Fig. 7)

b) the quadrangle the vertices and side bisectors of which belong to $\mathbf{K}$ and $\mathbf{L}$ respectively.

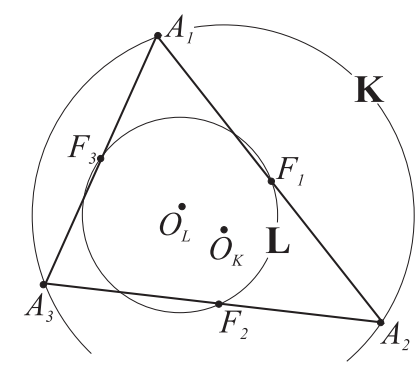

Fig. 7

Solution to Problem 4a). (Fig. 8)

The midpoints $F_{A}, F_{B}, F_{C}$ of the sides of the triangle $A B C$ constitute a triangle homothetic to $A B C$. The ratio of dilatation is $1 / 2$, the centre of dilatation is the common centre of gravity $S$ of the triangles. A dilatation with ratio $(-1 / 2)$ (i.e. a composition of a contraction with ratio $1 / 2$ followed by a central reflection) centered at $S$ maps the triangle $A B C$ onto $F_{A} F_{B} F_{C}$, the circle $\mathbf{K}$ onto $\mathbf{L}$ and the centre $O_{K}$ of $\mathbf{K}$ onto the centre $O_{L}$ of $\mathbf{L}$.

Thus one deduces that the constructibility of the triangle requires the radius of $\mathbf{L}$ to be half the radius of $\mathbf{K}$. If this condition is fulfilled then, as will be immediately apparent, any chord $A B$ of $\mathbf{K}$ the midpoint $F_{C}$ of which lies on $\mathbf{L}$ is part of an appropriate triangle. Indeed, starting from given circles $\mathbf{L}$ and $\mathbf{K}$, the point $S$ can be constructed as the trisection point of the segment $O_{L} O_{K}$, lying nearer to $O_{L}$. The dilatation of ratio $(-2)$ centered at $S$ maps $\mathbf{L}$ onto $\mathbf{K}$. Let $F_{C}$ be the image of $C$ under this transformation. Point $S$ is necessarily the centre of gravity of the triangle $A B C$ since it is a trisection point of the median $C F_{C}$. If one now contracts $\mathbf{K}$ in the ratio $(-1 / 2)$ through $S$, then one arrives at the circle $\mathbf{L}$ and the points $A, B$ are taken to the midpoints $F_{A}, F_{B}$ both lying on $\mathbf{L}$.

Solution to Problem 4b):

As a first step the conditions for constructibility will be clarified. These are based on the following well-known statements: 


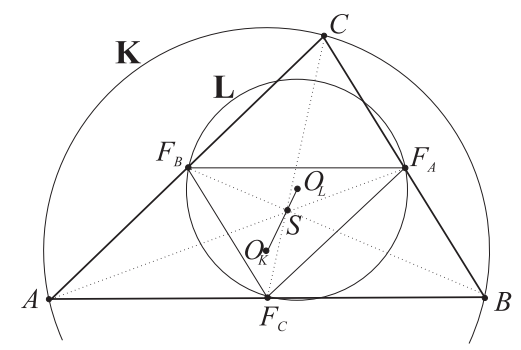

Fig. 8

Lemma 1. Midpoints of the sides of a general quadrilateral are vertices of a parallelogram the sides of which are parallel to the diagonals of the quadrilateral.

Lemma 2. The sum of the squares of the sides of any parallelogram equals the sum of the squares of its diagonals.

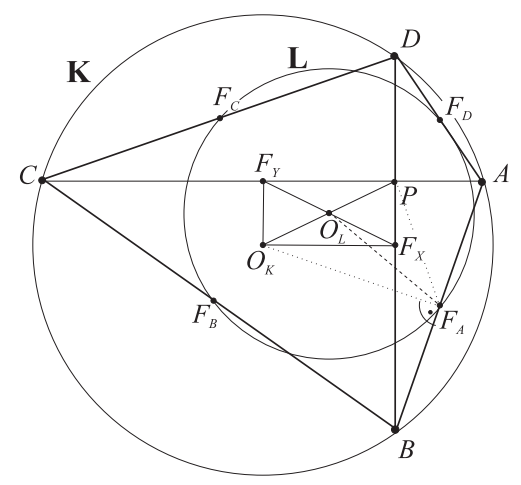

Fig. 9

Lemma 1 permits us to conclude (Fig. 9) that the midpoints of the quadrilateral ABCD we are looking for constitute a rectangle, since a rectangle is the only parallelogram which can be inscribed in a circle. It is also clear that diagonals $A C$ and $B D$ are perpendicular to each other. From this we deduce that $A P B \angle=90^{\circ}$ and the reverse of Thales'-theorem ensures that $\overline{A F_{A}}=\overline{F_{A} P}$.

Now we assert that the midpoint of the segment $O_{K} P$ is just $O_{L}$. For the proof, in addition to the rectangle $F_{A} F_{B} F_{C} F_{D}$, we take into consideration the parallelogram of the midpoints $F_{Y}, F_{C}, F_{X}, F_{A}$ of the quadrilateral $A C D B$ (twisted on the figure), as well as the rectangle $O_{K} F_{Y} P F_{X}$ bounded by the perpendicular chords $A C, B D$ and their perpendicular bisectors. Now we prove that all of these quadrilaterals have $O_{L}$ as their centre. For the rectangle $F_{A} F_{B} F_{C} F_{D}$ this follows because its centre is the same as that of the circle circumscribed around it, for the parallelogram $F_{Y} F_{C} F_{X} F_{A}$ because its diagonal $F_{C} F_{A}$ is a diagonal of the previous rectangle too and, finally, for the rectangle $O_{K} F_{Y} P F_{X}$ since it shares the diagonal $F_{X} F_{Y}$ with the previous parallelogram. 
Let us now apply Lemma 2 to the parallelogram which has $O_{K} P$ as one of its diagonals and $F_{A} O_{L}$ as half of the other:

$$
2{\overline{F_{A} P}}^{2}+2{\overline{F_{A} O_{K}}}^{2}={\overline{O_{K} P}}^{2}+4{\overline{O_{L} F_{A}}}^{2} .
$$

Here $\overline{O_{L} F_{A}}=R, \overline{O_{K} P}=2 d$ and in the right-angled triangle $A F_{A} O_{K}$ the relation ${\overline{F_{A} O_{K}}}^{2}=r^{2}-{\overline{A F_{A}}}^{2}$ is valid. We have, therefore,

$$
2{\overline{F_{A} P}}^{2}+2 r^{2}-2{\overline{A F_{A}}}^{2}=4 d^{2}+4 R^{2} .
$$

We already know that $\overline{F_{A} P}=\overline{A F_{A}}$, from which the exact condition for constructibility is obtained:

$$
2\left(R^{2}+d^{2}\right)=r^{2} .
$$

The construction itself is very simple once condition (2) is satisfied. We assert that any chord $A B$ of $\mathbf{K}$ whose midpoint $F_{A}$ belongs to the circle $\mathbf{L}$ may be completed up to an appropriate quadrilateral provided one minor further criterion is fulfilled, namely $\mathbf{L}$ must not be a circle of radius $r / 2$, containing the centre of $\mathbf{K}$.

We choose a point $P$ for which the midpoint of the segment $O_{K} P$ coincides with $O_{L}$. The construction then consists in simply drawing, from points $A$ and $B$, the chords $A C$, $B D$ of $\mathbf{K}$ through $P$. Our supplementary condition is necessary only to prevent $P$ from lying on $\mathbf{K}$ thus making the procedure possible, with points $A, B, C, D$ all different. It remaines only to prove that the midpoints of the sides of the quadrilateral obtained are on the circle $\mathbf{L}$.

Equations (1) and (2) are now valid by construction. Hence $\overline{F_{A} P}=\overline{A F_{A}}$ and $A P B \angle=$ $90^{\circ}$. Since the perpendicular bisectors of $A P, P C$ and $A C$ contain the points $F_{A}, F_{B}$ and $O_{K}$ respectively, the midpoint $O_{L}$ of $O_{K} P$ lies on the perpendicular bisector of $F_{A} F_{C}$ which is parallel to $A C$. The point $F_{B}$, therefore, belongs to $\mathbf{L}$ as required. The proof for the other midpoints is analogous.

These problems also have an infinite construction process which we shall call the Ponzag process (PONcelet + zig-ZAG) in their background. It is based on the circle $\mathbf{K}$ and object $\mathbf{L}$ (which may be either a circle or a straight line) and starts from the chord $A_{1} A_{2}$ whose bisector $F_{1}$ lies on $\mathbf{L}$. The sequence of points $\left\{A_{n}\right\}$ is generated by repeated application of the following construction:

Let $A_{n}$ be the point of intersection of two circles the first of which is $\mathbf{K}$ and the second is the image of $\mathbf{L}$ under the dilatation of ratio 2 centered at $A_{n-1}$. Choose for $A_{n}$ the point of intersection which is different from $A_{n-2}$. If no such point exists then put $A_{n}=A_{n-2}$.

The midpoint $F_{n-2}$ of the segment $A_{n-2} A_{n-1}$ belongs to $\mathbf{L}$, hence $A_{n-2}$ will certainly be an intersection point. Therefore, the construction cannot fail due to the absence of any intersection point. The uniqueness of the process is ensured if the doubly magnified image of $\mathbf{L}$ does not coincide with $\mathbf{K}$, i.e. if the centre of $\mathbf{K}$ does not lie on $\mathbf{L}$ and the latter's radius is not just half of the radius of the former.

The questions stated in Problem 4 now reduce to the selection of those points $A_{1}$ which give rise to 3 or 4 -step periodic processes. As it turns out the answer is similar to the Zig-zag theorem. 
The Ponzag theorem. Let $\mathbf{K}$ be a circle and $\mathbf{L}$ an object (a circle or a straight line) which does not contain the centre of $\mathbf{K}$. If the Ponzag process based on them is nperiodic when started from some given chord, then it remains of periodicity $n$ for any other choice of the initial chord.

Geometers of the XIX. century would have formulated the theorem somewhat differently: if there exists an n-gon whose vertices lie on the circle $\mathbf{K}$ and the midpoints of the sides lie on another circle (or straight line) $\mathbf{L}$, then an infinite number of such $n$-gons exist. ${ }^{2}$

The Ponzag theorem will be proved in Section 4, where it will be traced back to the Zig-zag theorem.

Let us now express our results in algebraic form. A Ponzag configuration of two circles is characterized by the radii $r, R$ of the circles $\mathbf{K}$ and $\mathbf{L}$ and the distance $d$ between their centres. The corresponding Ponzag configuration will be denoted by $\operatorname{PZ}(r, R, d)$. We have already found polynomials $p z_{3}, p z_{4}$, satisfying periodicity

$$
\begin{aligned}
& p z_{3}(r, R, d)=r-2 R \\
& p z_{4}(r, R, d)=r^{2}-2\left(R^{2}+d^{2}\right) .
\end{aligned}
$$

Notice that when $\mathbf{L}$ is a straight line then the periodicity is necessarily 4 -step because if $A_{1} A_{2}$ is a chord of $\mathbf{K}$ the midpoint of which lies on $\mathbf{L}$, then parallels to $\mathbf{L}$ through $A_{1}$ and $A_{2}$ cut out of $\mathbf{K}$ a trapezium the median of which is just $\mathbf{L}$. The diagonals of this trapezium together with its sides form a twisted Ponzag quadrilateral (see the dotted quadrilateral on Fig. 14).

\section{Polygons which have both inscribed and circumscribed circles}

Problems 5a) and 5b) below are classic having already been dealt with by Euler. Therefore, in this section, we will concentrate mainly on history rather than giving proofs. This is all the more justifiable since in the next section new proofs for these old problems will be proposed.

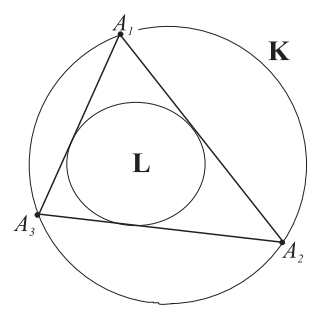

Fig. 10

2) I failed to find any reference to the case $n>3$. 
Problem 5. Let $\mathbf{K}$ and $\mathbf{L}$ be two circles in a plane. Construct

a) the triangle (Fig. 10)

b) the quadrangle, the vertices of which lie on $\mathbf{K}$ and the sides of which are tangent to $\mathbf{L}$.

It is not difficult to establish the recursive process (Poncelet construction) suitable to this problem. If we start from chord $A_{1} A_{2}$ of $\mathbf{K}$ tangent to $\mathbf{L}$, then the sequence of points $\left\{A_{n}\right\}$ is obtained by the repeated application of the following elementary step:

Choose $A_{n}$ to be the point of intersection of $\mathbf{K}$ with the tangent to $\mathbf{L}$ which starts from $A_{n-1}$; if possible avoid the choice of $A_{n-1}$ for $A_{n}$ and $A_{n-2} A_{n-1}$ for the tangent from $A_{n-1}$.

The question is how to select those "tangential chords" $A_{1} A_{2}$ starting from which the sequence $\left\{A_{n}\right\}$ becomes 3- or 4-step periodic.

The condition for constructibility of a triangle follows from a theorem due to Euler which states that the radii $R$ and $r$ of the circumscribed and inscribed circles of a triangle and the distance $d$ between their centres satisfy the relation

$$
R^{2}-d^{2}=2 R r .
$$

A beautiful proof of this theorem is found in the work of Coxeter and Greitzer [Theorem 2.1.2] referred to above.

Problem 5, however, does not require the circle $\mathbf{L}$ to be the inscribed circle, it may be an escribed one as well (Fig. 11). A minor modification of the proof leads to the formula

$$
\left|R^{2}-d^{2}\right|=2 R r
$$

which encompasses the general case.

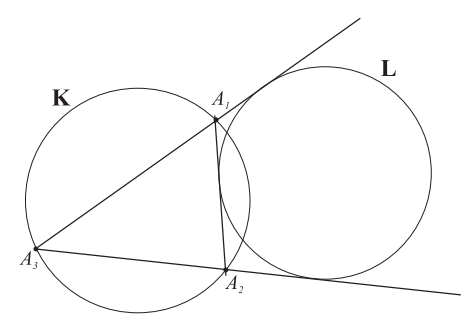

Fig. 11

It is also true that if this relation is taken for granted then any "tangential chord" may be completed up to a triangle which is both inscribed into $\mathbf{K}$ and tangent to $\mathbf{L}$. The proof of this statement will be given in the next section from a rather different angle.

Euler himself made efforts to generalize his formula from triangles to quadrangles but without success. The desired relation was finally found in 1797 by his former pupil Fuss in Petersburg ([1], p. 33 and [2]):

$$
2 r^{2}\left(R^{2}+d^{2}\right)=\left(R^{2}-d^{2}\right)^{2} .
$$


An elementary proof of this formula can be found in the book of Dörrie [9]. To the surprise of the present author the formula of Fuss does not cover a certain class of twisted quadrangles (see Fig. 14). In Section 4 the formula (4) will be derived and properly extended. It will also be shown that whenever it is satisfied, any tangential chord of $\mathbf{K}$ may be completed up to a quadrangle inscribed into $\mathbf{K}$ and tangent to $\mathbf{L}$.

For polygons with an increasing number of vertices the problem becomes more and more difficult: for $n$-gons of $n=5,6,7,8$ Fuss was able to give formulae only for the special case when the polygons were symmetric with respect to the line of centres of the circles ([3], [7]).

The French engineer-mathematician J.V. Poncelet in 1817 [4] published a striking theorem which in the terminology of the present work may be formulated as follows: Let $\mathbf{K}$ and $\mathbf{L}$ be two nondegenerate conics which are not tangent to each other. If a tangential chord exists which gives rise to an n-step periodic Poncelet process then the process remains $n$-step periodic for any initial tangential chord.

The proof of the theorem is beyond the scope of the present work. The interested reader is referred to the works of Poncelet ([5], [6]) where a purely geometric proof, leading to an even more general theorem, is given. The same route in more elegant steps is described by Lebesgue in [14] and also by Berger, in his readily available well illustrated book [16], published in several languages.

Jacobi found a rather peculiar proof of the theorem by relating it to an integration problem. His original writings [7] permit us to follow his novel approach but the essence of his proof is easier to understand via the short article of Shen [22].

It was Cayley who, also working with integrals like Jacobi, discovered those polynomials whose zeros express periodicity. The polynomials of Cayley depend on the coefficients in the equations of the conics $\mathbf{K}$ and $\mathbf{L}$. His proof was translated by Lebesgue [14] into a geometric and by Griffith and Harris [10] into a modern algebraic-geometric language. More recently new results concerning Poncelet's theorem were communicated by Barth and Michel [20]. In particular, they determined how many elements in a pencil of conics form Poncelet configurations with a given conic. They were also able to identify those pairs of conics the first of which circumscribes the n-gon tangent to the second while, at the same time, the second circumscribes the m-gon tangent to the first.

We now formulate the closure theorem of Cayley which will be employed in the next section. As will be seen, Problems 1, 2 originate in this theorem.

Let the conics $\mathbf{K}$ and $\mathbf{L}$ be characterized by their matrices $K, \Lambda$. Form the matrix $t K+\Lambda$. The square root of the determinant of this matrix may be expanded into a power series in $t$ :

$$
\sqrt{\operatorname{det}(t K+\Lambda)}=A_{0}+A_{1} t+A_{2} t^{2}+\cdots
$$

The Poncelet construction will be n-periodic iff

$$
\left|\begin{array}{ccc}
A_{2} & \cdots & A_{m+1} \\
\cdots & \cdots & \cdots \\
A_{m+1} & \cdots & A_{2 m}
\end{array}\right|=0 \text { if } n=2 m+1 \text { or }\left|\begin{array}{ccc}
A_{3} & \cdots & A_{m+1} \\
\cdots & \cdots & \cdots \\
A_{m+1} & \cdots & A_{2 m-1}
\end{array}\right|=0 \text { if } n=2 m \text {. }
$$

In the spirit of the present work the above determinants should be denoted by $p_{n}$. They vanish whenever the configuration is $P_{n}$. 


\section{Connections}

In this section the equivalence of the Zig-zag, the Ponzag and the Poncelet theorems will be demonstrated. Recently Barth and Bauer published an extensive study of the subject. They were able to relate several Poncelet-type theorems to a common origin [22] which is a spatial version of the Poncelet theorem discovered by Weyr ([12], [24]). Below we make an attempt to present the interconnections of the aforementioned three theorems as transparently as possible, using a rather more elementary approach. The equivalence will be established in the following order:

$$
\text { Zig-zag } \Longrightarrow \text { Ponzag } \Longrightarrow \text { Poncelet } \Longrightarrow \text { Zig-zag. }
$$

\section{Ponzag follows from Zig-zag.}

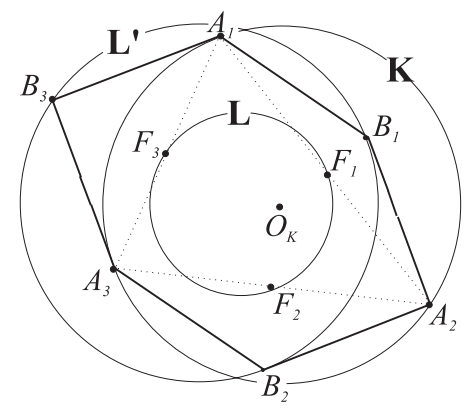

Fig. 12

Proof. Consider Fig. 7 from Problem 4. Reflect the centre $O_{K}$ of the circle $\mathbf{K}$ centrally with respect to the points of $\mathbf{L}$ (Fig. 12). The image-manifold $\mathbf{L}^{\prime}$ will be a circle or a straight line, depending on what $\mathbf{L}$ is. Alternatively, $\mathbf{L}^{\prime}$ may also be considered as the image of $\mathbf{L}$ under the dilatation of ratio 2 centered at $O_{K}$.

Let the images of $O_{K}$ under central reflection with respect to the midpoints $F_{1}, F_{2}, F_{3}$ be the points $B_{1}, B_{2}, B_{3}$ respectively. Since the quadrilateral $O_{K} A_{i} B_{i} A_{i+1}$ is a rhombus, $A_{1} B_{1} A_{2} B_{2} A_{3} B_{3} \cdots$ is a periodic zig-zag between $\mathbf{K}$ and $\mathbf{L}^{\prime}$. It may be seen that our method is quite a general one: by a twofold dilatation of $\mathbf{L}$ centered at $O_{K}$ we have managed to relate a Ponzag configuration, belonging to $\mathbf{K}$ and $\mathbf{L}$, to a Zig-zag configuration between $\mathbf{K}$ and $\mathbf{L}^{\prime}$ in such a manner that the $P Z_{n}$ configurations correspond precisely to the $Z_{n}$ configurations.

\section{Poncelet follows from Ponzag.}

We will show that the two-circle version of the Poncelet theorem follows from the Ponzag theorem; our proof will not cover the general case. This is not a serious limitation however since the general theorem of Poncelet follows from this special version - in his proof Poncelet himself followed the same route.

Proof. Consider Fig. 10 from Problem 5. Perform an inversion with respect to the circle $\mathbf{L}$ (Fig. 13). The image of $\mathbf{K}$ under this inversion will be the figure $\mathbf{K}^{\prime}$ which is either 


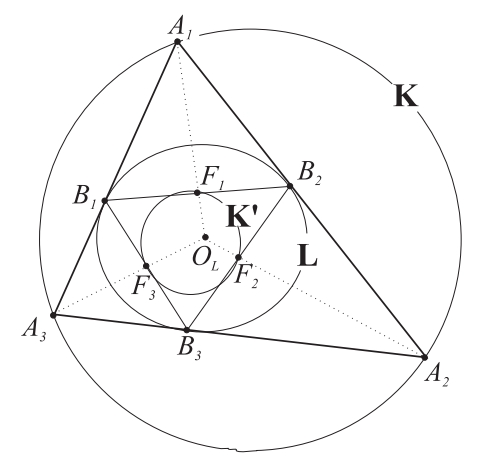

Fig. 13

a circle or a straight line. We assert that the image of the point $A_{1}$ will be the midpoint $F_{1}$ of the segment, connecting the contact points $B_{2}$ and $B_{1}$ of the tangents $A_{1} A_{2}$ and $A_{1} A_{3}$. This follows from the symmetry which forces the points $O_{L}, F_{1}, A_{1}$ to lie on a common straight line and the triangles $O_{L} A_{1} B_{1}$ and $O_{L} B_{1} F_{1}$ to be similar since they are of right angle and have at $O_{L}$ a common angle. Hence $\overline{O_{L} A_{1}} / \overline{O_{L} B_{1}}=\overline{O_{L} B_{1}} / \overline{O_{L} F_{1}}$ i.e. $\overline{O_{L} A_{1}} \cdot \overline{O_{L} F_{1}}=r^{2}$.

Now it is seen that the inversion of the circle $\mathbf{K}$ with respect to $\mathbf{L}$ permits us to associate a Ponzag configuration between $\mathbf{K}$ and $\mathbf{L}^{\prime}$ to a Poncelet configuration belonging to the circles $\mathbf{K}$ and $\mathbf{L}$, and moreover, the configurations $P Z_{n}$ correspond exactly to the $P_{n}$ configuration.

In order to illustrate this correspondence in an elementary way we now compute, from the polynomials $p z_{3}$ and $p z_{4}$, the polynomials $p_{3}, p_{4}$, i.e. the relations occurring in Euler's and Fuss' theorems. As a preliminary step we have to express in terms of $R$ and $d$ the radius $R^{\prime}$ of $\mathbf{K}^{\prime}$ and the distance $d^{\prime}$ of its centre from the centre of inversion $O_{L}$. This is not a difficult task since, owing to symmetry, we may confine ourselves to the determination of the transformed positions of those points of $\mathbf{K}^{\prime}$ only which lie on the line through the centres of the circles:

$$
\begin{aligned}
& R^{\prime}=\frac{1}{2}\left|\frac{r^{2}}{d-R}-\frac{r^{2}}{d+R}\right|=r^{2}\left|\frac{R}{d^{2}-R^{2}}\right|, \\
& d^{\prime}=\frac{1}{2}\left|\frac{r^{2}}{d-R}+\frac{r^{2}}{d+R}\right|=r^{2}\left|\frac{d}{d^{2}-R^{2}}\right| .
\end{aligned}
$$

If these expressions are now substituted into $p z_{3}$ and $p z_{4}$, then we obtain formulae equivalent to (3) and (4). One must, however, take into account the fact that $\mathbf{K}^{\prime}$ may turn out a straight line as well and this is just a 4-step Ponzag configuration (Fig. 14). This happens when $\mathbf{K}$ passes through the centre of $\mathbf{L}$ i.e. when $d=R$. In this case twisted quadrilaterals akin to the zig-zag configuration of Problem 2 arise whose sides are tangential chords. The polynomials promised earlier are:

$$
\begin{aligned}
& p_{3}(r, R, d)=\left(R^{2}-d^{2}\right)^{2}-4 R^{2} r^{2} \quad \text { and } \\
& p_{4}(r, R, d)=(R-d)\left[\left(R^{2}-d^{2}\right)^{2}-2 r\left(R^{2}+d^{2}\right)\right] .
\end{aligned}
$$




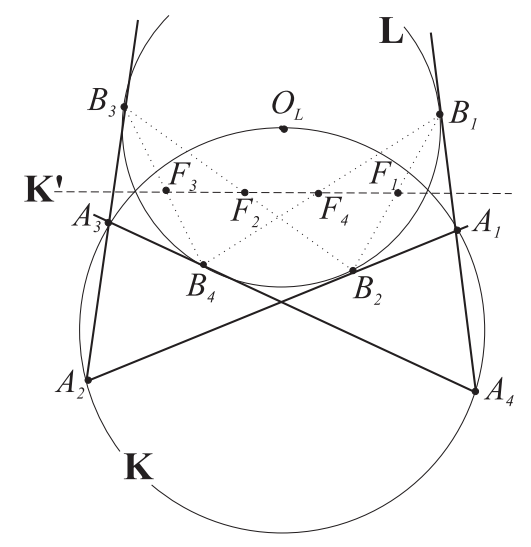

Fig. 14

\section{Zig-zag follows from Poncelet.}

Consider Fig. 1, from Problem 1. According to the Zig-zag process, the circles of radius $\rho$ around $B_{1}, B_{2}$ and $B_{3}$ intersect the circle $\mathbf{K}_{\mathbf{A}}$ at the pairs of points $\left(A_{1}, A_{2}\right),\left(A_{2}, A_{3}\right)$ and $\left(A_{3}, A_{1}\right)$. In Fig. 15 , in addition to $B_{1}, B_{2}, B_{3}$, a further point $B$ on the circle $\mathbf{K}_{\mathbf{B}}$ was selected, around which a circle of radius $\rho$ was drawn. The points of intersection of this circle with $\mathbf{K}_{\mathbf{A}}$ were connected by a straight line.

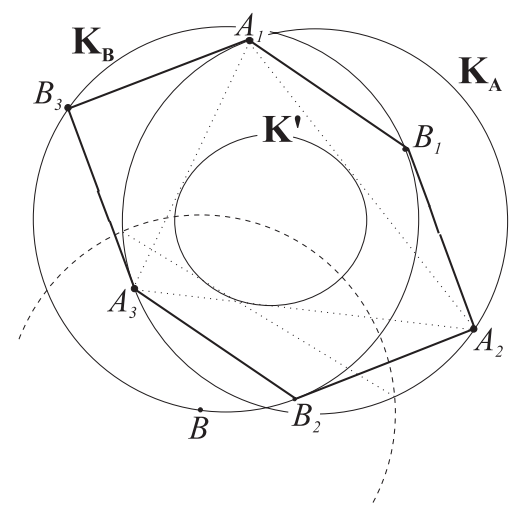

Fig. 15

Imagine now that $B$ moves along $\mathbf{K}_{\mathbf{B}}$. If all the straight lines corresponding to different positions of $B$ were tangent to some conics $\mathbf{K}^{\prime}$, then the Zig-zag construction algorithm between $\mathbf{K}_{\mathbf{A}}$ and $\mathbf{K}_{\mathbf{B}}$ would be equivalent to the Poncelet construction between $\mathbf{K}_{\mathbf{A}}$ and $\mathbf{K}^{\prime}$.

The existence of $\mathbf{K}^{\prime}$ - and thereby the reduction of the Zig-zag theorem to the Poncelet theorem - will be demonstrated for the general spatial configuration i.e. when the circles $\mathbf{K}_{\mathbf{A}}, \mathbf{K}_{\mathbf{B}}$ are not confined to a common plane. 
The following lemma will be needed:

Lemma. Let $\Gamma$ be a cone in euclidean space the vertex of which is $O$. Consider the set $\mathcal{M}$ of planes perpendicular to the generators of $\Gamma$ and having the arbitrarily chosen point $P$ common. Then $M$ will be the set of tangent planes to some cone $\Gamma^{\prime}$ with vertex $P$.

Here we assume the validity of this lemma without proof but notice that its content is nothing but reciprocation ([15] Chapter 6) ${ }^{3)}$.

Consider the sphere $\mathbf{G}_{\mathbf{B}}$ of radius $\rho$ around the point $B$ of $\mathbf{K}_{\mathbf{B}}$ and some sphere $\mathbf{G}$ which contains the circle $\mathbf{K}_{\mathbf{A}}$ (Fig. 16). Consider also the intersection plane of $\mathbf{G}_{\mathbf{B}}$ and $\mathbf{G}$ (rather than the straight line connecting the points of intersection of $\mathbf{G}_{\mathbf{B}}$ and $\mathbf{K}_{\mathbf{A}}$ ) which, owing to its similarity to the notion of the radical axis, will be called the radical plane. Denote by $P$ the point of intersection of this plane with the symmetry axis $\mathbf{t}$ of the circle $\mathbf{K}_{\mathbf{B}}$ (Fig. 17). The existence of a point of intersection is guaranteed if $\mathbf{G}$ is chosen so that its centre $O$ lies outside the plane of $\mathbf{K}_{\mathbf{B}}$. The point $P$ has the same power with respect to $\mathbf{G}$ and all the circles of radius $\rho$ whose centres are on $\mathbf{K}_{\mathbf{B}}$. Hence, for any choice of the point $B$ on $\mathbf{K}_{\mathbf{B}}$ the intersection plane of the spheres $\mathbf{G}_{\mathbf{B}}$ and $\mathbf{G}$ will necessarily pass through $P$.

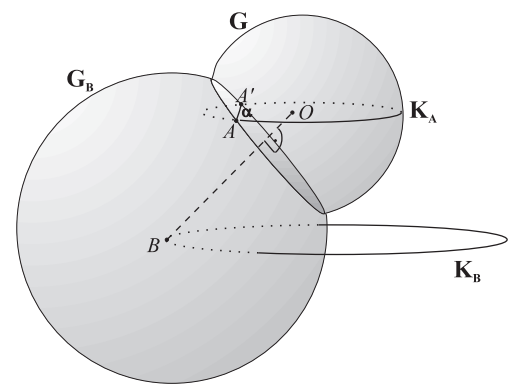

Fig. 16

On the other hand, the radical plane of $\mathbf{G}_{\mathbf{B}}$ and $\mathbf{G}$ is nothing but the plane through $P$ orthogonal to the straight line $O B$. If now we apply our lemma to the cone of vertex $O$ and base line $\mathbf{K}_{\mathbf{B}}$ on the one hand and the point $P$ on the other, then we arrive at the promised conic $\mathbf{K}^{\prime}$ in the plane of $\mathbf{K}_{\mathbf{A}}$.

At first sight the proof seems to fail when only one of the objects $\mathbf{K}_{\mathbf{A}}, \mathbf{K}_{\mathbf{B}}$ is a circle. When, for example, $\mathbf{K}_{\mathbf{A}}$ is a straight line, then the lines connecting the points of intersection of the spheres $\mathbf{G}_{\mathbf{B}}$ of radii $\rho$ around the points of $\mathbf{K}_{\mathbf{B}}$ with $\mathbf{K}_{\mathbf{A}}$, always degenerate into the straight line $\mathbf{K}_{\mathbf{A}}$ itself. But it remains possible to apply an inversion with respect to a

3) Let $\Sigma$ be any plane, not containing $O$. A map $\phi$ can be defined which makes the points of $\Sigma$ correspond to lines of the same plane: if $Q$ is any point of $\Sigma$ then let $\phi(Q)$ be the intersection line of $\Sigma$ with the plane, passing through $P$ and perpendicular to $O Q$.

It can be proved that this map is the reciprocation with respect to a, possibly imaginary, circle. This circle is the intersection line of $\Sigma$ with the Thales sphere of segment $O P$. 


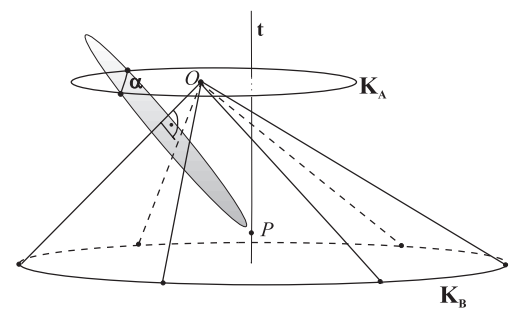

Fig. 17

sphere orthogonal to all the spheres $G_{B}$ under which $\mathbf{K}_{\mathbf{A}}$ transforms into a circle, while the spheres of radius $\rho$ mentioned above remain unchanged. After this transformation has been performed the previous argument becomes applicable again.

What happens when both $\mathbf{K}_{\mathbf{A}}$ and $\mathbf{K}_{\mathbf{B}}$ are straight lines? Even if they are situated in space this is a much simpler problem than the theorem of Poncelet so there is no reason to trace it back to the latter. The planar case has already been thoroughly discussed in Problem 3. The spatial version turns out to be a simple corollary to $\mathrm{it}^{4)}$.

The argument above proves that to every Zig-zag configuration there corresponds some Poncelet configuration. Since the closure conditions for the latter have already been clarified by Cayley it becomes - in principle - possible to write down the closure conditions of the Zig-zag configurations too. I computed, with the aid of the Mathematica software, the corresponding low $n$ polynomials for the planar configuration of the circles. The first three polynomials are ${ }^{5)}$ :

$$
\begin{aligned}
& z_{2}(r, R, d, \rho)=d^{2}+\rho^{2}-r^{2}-R^{2} \\
& z_{3}(r, R, d, \rho)=\left(d^{2} \rho^{2}-r^{2} R^{2}\right)^{2}-\left(d^{2}+\rho^{2}-r^{2}-R^{2}\right)^{2} r^{2} R^{2} \\
& z_{4}(r, R, d, \rho)=(d \rho-r R) \cdot\left\{2\left(d^{2} \rho^{2}-r^{2} R^{2}\right)^{2}-\left(d^{2} \rho^{2}+r^{2} R^{2}\right)\left(d^{2}+\rho^{2}-r^{2}-R^{2}\right)^{2}\right\} .
\end{aligned}
$$

For the planar case of a circle and a straight line the following polynomials are obtained:

$$
\begin{aligned}
& z_{2}^{\prime}(r, \Delta, \rho)=\Delta \\
& z_{3}^{\prime}(r, \Delta, \rho)=\left(r^{2}-\rho^{2}\right)^{2}-4 r^{2} \Delta^{2} \\
& z_{4}^{\prime}(r, \Delta, \rho)=(r-\rho) \cdot\left\{\left(r^{2}-\rho^{2}\right)^{2}-\Delta^{2}\left(r^{2}+\rho^{2}\right)\right\} .
\end{aligned}
$$

These formulae served as the starting point for the first two problems.

The explicit forms of the lowest order polynomials $z_{2}, z_{3}, z_{4}$ betray unexpected symmetries which go far beyond the natural symmetry with respect to the interchange of

4) To show this, one only has to project the whole configuration onto a plane parallel to both lines. The "zigs" remain equal after this projection and a planar zig-zag configuration is obtained.

5) A 2-step periodic process is 4-step periodic too. Therefore, for the polynomials derived from Cayley's formula $z_{2} \mid z_{4}$. Our $z_{4}$ and $z_{4}^{\prime}$ are primitive Zig-zag polynomials in the sense that the corresponding second order polynomials have already been factored out of them. 
the radii $r$ and $R$ of the circles. However, there seems to be no compelling reason for symmetry with respect to $d$ and $\rho$. It is even more surprising that for even periodicities the pair $r, R$ is interchangeable with the pair $d, \rho$. These observations based on the lowest order polynomials turn out to be generally valid and may be summarized in the following theorems:

First duality theorem $(d \Longleftrightarrow \rho)$.

If in a planar Zig-zag configuration of periodicity $n$ we retain the radii of the circles but for the new distance between their centres choose the length of the "zag", and for the new "zag" the original distance between the centres, then the new configuration remains of periodicity $n$.

Second duality theorem $(r \Longleftrightarrow \rho, \quad R \Longleftrightarrow d)$.

Given a planar Zig-zag configuration of periodicity $n$, if we simultaneously interchange the radius of one of the circles and the length of the zag on the one hand, and the radius of the other circle with the distance between the centres on the other, then for even $n$ we obtain an n-periodic and for odd $n$ a $2 n$-periodic configuration.

Note added in proof. For the proof of the above duality theorems and the generalization of the Zig-zag Theorem to Euclidean, spherical and hyperbolic $n$-spaces see [29]. After the present article was accomplished Prof. H. Stachel drew my attention to the important papers [25], [26], [27], [28]. In particular, the Zig-zag Theorem for two coplanar circles was first published by O. Bottema in Ref [25].

Acknowledgement. My thanks to F. Pogács for drawing my attention to [17]. I am grateful to B. Csikós for his constant interest and valuable suggestions and to Thalia Towsend for help in English. This article would not have been written without the encouragement of my father and without the support of Lauder School, my former place of work.

\section{References}

[1] V.I. Lisenko, N.I. Fuss, Nauka, Moscow 1960.

[2] N.I. Fuss, De quadrilaterlis quibus circulum tam inscribere quam circumscribere licet; NAASP 1792 (Nova Acta), t.X, (1797), 103-125 (14. VIII 1794).

[3] N.I. Fuss, De polygonis simmetrice irregularibus calculo simul inscriptis et circumscriptis; NAASP 1795-1796 (Nova Acta), t.XIII, (1802), 168-189 (19. IV 1798).

[4] Annales de Mathématiques de Nîmes 1817.

[5] J.V. Poncelet, Traité des propriétés projectives des figures, t.I; Paris 1865, (first ed. in 1822).

[6] J.V. Poncelet, Applications d'analyse et de géométrie, t.I; Paris, Mallet-Bachelier, Imprimeur-Libraire, 1862.

[7] C.G.J. Jacobi, Über die Anwendung der Elliptischen Transcendenten auf ein bekanntes Problem der Elementargeometrie; Crelle Journal für die reine und angewandte Mathematik; 3 (1828), 376, and in: Gesammelte Werke, 278-293.

[8] A. Cayley, Note on the geometrical representation of the integral $\int d x \div \sqrt{(x+a)(x+b)(x+c)}$; Philosophical Magazine, vol. V (1853), 99-102.

Note on the Porism of the in-and-circumscribed polygon; Philosophical Magazine, vol. VI (1853), 376377 . 
Correction of two theorems relating to the porism of the in-and-circumscribed polygon; Philosophical Magazine, vol. VI (1853), 376-377.

Developments on the porism of the in-and-circumscribed polygon; Philosophical Magazine, vol. VII (1854), 339-345.

(See papers 113, 115, 116, 128 in Collected Works of Cayley)

[9] H. Dörrie, Triumph der Mathematik; Würzburg, Physica-Verlag, 1958, Problem 39.

[10] P.A. Griffiths and S. Harris, On Cayley's explicit solution of Poncelet's porism; L'Enseignement Math. 24 (1978), 31-40.

[11] P.A. Griffiths, Variations on a Theorem of Abel; Inventiones mathematicae 35 (1976), 321-390.

[12] P.A. Griffiths and J.Harris, A Poncelet theorem in space; Commentarii Mathematici Helvetici 52 (1977), $145-160$.

[13] H.S.M. Coxeter and S.L. Greitzer, Geometry Revisited; Random House, New York 1967.

[14] H. Lebesgue, Les coniques, Ch. IV; Gauthier-Villars, Paris 1955.

[15] W.L. Black, H.C. Howland and B. Howland, A theorem about zigzags between two circles; Amer. Math. Monthly 81 (1974), 754-757.

[16] M. Berger, Géométrie, Sect. 16.6; CEDIC/Fernand Nathan, Paris 1977.

[17] H. Walser, Ein Schliessungssatz der Elementargeometrie, Elemente der Mathematik; 43 Nr. 6 (1988), 161-169.

[18] Supplement Nr. 1/1997 to Russian journal KVANT - MATEMATIKA, problem 190.

[19] N.B. Vasiliev, Sequential jumps; KVANT Nr.11 (1973), 25-31.

[20] W. Barth and Th. Bauer, Poncelet Theorems; Expositiones Mathematicae 14 (1996), 125-144.

[21] W. Barth and J. Michel, Modular curves and Poncelet polygons; Matematische Annalen 295 (1993), $25-49$.

[22] A. Shen ed., Mathematical Entertainments, Poncelet Theorem Revisited; The Mathematical Intellingencer, 20 Nr.4 (1998), 31.

[23] A. Hraskó, Theorem of Poncelet and related problems, Eötvös Roland University Budapest, Thesis, 1991.

[24] E. Weyr, Über einige Sätze von Steiner und ihren Zusammenhang mit der zwei und zweigliedrigen Verwandtschaft der Grundgebilde ersten Grades; Crelle Journal für die reine und angewandte Mathematik, Bd. 71 (1870), 18-28.

[25] O. Bottema, Ein Schliessungssatz für zwei Kreise; Elem. Math. 20 (1965), 1-7.

[26] F. Hohenberg, Ein Schliessungssatz über gleichseitige Polygone, deren Ecken abwechselnd auf zwei Kreisen liegen; Sb. Österreichische Akademie der Wissenschaften 186 (1977), 281-300.

Geschlossene gleichseitige Polygone, deren Ecken abwechselnd einem Kreis und einer Geraden angehören; Sb. Österr. Akad. Wiss. 188 (1979), 143-156.

Besondere gleichseitige Zwölfecke, die sich aus einem Schliessungssatz ergeben; Sb. Österr. Akad. Wiss. 188 (1979), 157-166.

Gleichseitige Polygone, deren Ecken abwechselnd auf zwei Geraden liegen; Sb. Österr. Akad. Wiss. 188 (1979), 385-405.

[27] F. Hohenberg, Eine besondere Art gleichseitiger Sechsecke; Elem. Math. Bd 32/3 (1977), 64-67.

[28] W. Wunderlich, Mechanisms Related to Poncelet's Closure Theorem, Mechanism and Machine Theory, Vol. 16 (1981) No 6., 611-620.

[29] B. Csikós, A. Hraskó, Remarks on the Zig-zag Theorem, Periodica Mathematica Hungarica Vol. 39 (1999), to be published.

András Hraskó

Mihály Fazekas Secondary School

H-1141 Budapest, Tornóc u. 17,

Hungary

hraskoa@freemail.hu 